\title{
La constancia de la razón entre la longitud de madurez y la longitud asintótica en peces de la costa de Chile: Un meta-análisis
}

\author{
The constancy of the ratio of the length at maturity and asymptotic length in fish \\ from the coast of Chile: A meta-analysis
}

\section{Miguel Araya ${ }^{1,2}$ y Renzo Pepe-Victoriano ${ }^{3,4}$}

\author{
${ }^{1}$ Programa Doctorado en Ciencias Aplicadas mención Sistemas Marinos Costeros, Facultad de Recursos del Mar, \\ Universidad de Antofagasta, Antofagasta, Chile. maraya@uantof.cl \\ ${ }^{2}$ Departamento de Ciencias del Mar, Universidad Arturo Prat, Casilla 121, Iquique, Chile \\ ${ }^{3}$ Departamento de Biología, Facultad de Ciencias, Universidad de Tarapacá, Casilla 7-D, Arica, Chile \\ ${ }^{4}$ Centro de Recursos Naturales y Medio Ambiente, Facultad de Ciencias, Universidad de Tarapacá, Casilla 7-D, Arica, Chile
}

\begin{abstract}
There is evidence for interspecific correlations among population parameters in fish. Such correlations have been described from parameters including those produced through the von Bertalanffy model, natural mortality, age and length at maturity. The correlations have been used as practical tools to predict the dynamics and life history characteristics of understudied populations, and even to estimate maximum sustainable yield. The value of invariant proposed by Jensen (i.e., $L_{m} / L_{\infty}=0.66$ ) has not been tested for fish from the Eastern South Pacific: hence, we evaluated the value of invariant for Chilean fish resources to determine the average effect size, the homogeneity around the mean effect size, and variables which might moderate the effect size, if homogeneity does not exist. In order to examine this question we conducted a meta-analysis of studies examining J ensen's invariant $\left(L_{m} / L_{\infty}=0.66\right)$, where we collected a total of 148 invariant values from 10 fish families. Significant differences were detected in these parameters between fish species, so the analysis was conducted with data pooled at the family level. The weighted average obtained with the random effects model was 0.61 . The families Merlucciidae, Nototheniidae, Ophidiidae, Scombridae and Trachichthyidae showed a value of 0.66 . The average for the Bramidae was 0.69 , and for Carangidae, Clupeidae and Engraulidae was $0.46,0.56$ and 0.51 respectively. These results showed that the theoretical value of the invariant $L_{m} / L_{\infty}=0.66$ as proposed by Jensen, is not general to the commercially important fishes of the Eastern South Pacific.
\end{abstract}

Key words: Invariant, Beverton \& Holt, ichthyology

Resumen. - Existen evidencias de la correlación interespecífica de los parámetros poblacionales en peces. Es así, que se encuentran relaciones entre los parámetros de crecimiento del modelo de von Bertalanffy, mortalidad natural, edad y longitud de madurez. Estas relaciones han servido como herramientas prácticas para caracterizar la dinámica y la historia de vida de una población poco estudiada o estimar parámetros de vida que en otros casos son muy difíciles de obtener (e.g., mortalidad natural). Debido a que el valor de la razón entre la longitud de madurez y la longitud asintótica en peces $\left(\mathrm{L}_{\mathrm{m}} / \mathrm{L}_{\infty}=0,66\right)$ propuesto por J ensen no ha sido probado para peces de la costa de Chile, se evaluó el valor de esta constancia o invariante, entendiéndose como una característica que no cambia en la historia de vida de los animales. Se estimó el tamaño del efecto medio (invariante) y su homogeneidad, además de las variables que pueden estar moderando el tamaño del efecto. Con el fin de responder a las preguntas se realizó un meta-análisis de la constancia $L_{m} / L_{\infty}=0,66$, se recopiló 148 valores de la invariante correspondiente a 10 familias de peces. Se encontraron diferencias significativas en estos parámetros entre especies de peces, por lo que los análisis se realizaron agrupando a nivel de familia. El valor de la media ponderado con el modelo de efectos aleatorios fue de 0,61 . Cinco familias contienen el valor 0,66 siendo estas Merlucciidae, Nototheniidae, Ophidiidae, Scombridae y Trachichthyidae. La media de la familia Bramidae es de 0,69 y Carangidae, Clupeidae y Engraulidae fue de 0,46; 0,56 y 0,51 , respectivamente, por debajo del valor de la invariante 0,66 . Finalmente, se desprende que el valor teórico $L_{m}$ $/ L_{\infty}=0,66$ propuesto por J ensen no es generalizable para todas las especies de peces comerciales del Pacífico sudeste.

Palabras clave: Invariante, Beverton \& Holt, ictiología

\section{INTRODUCCIÓN}

Evidencias de la correlación interespecífica de los parámetros poblacionales en peces fueron otorgadas por
Beverton \& Holt (1959) al relacionar el patrón de crecimiento (parámetros del modelo de von Bertalanffy), 
la estrategia reproductiva (edad y longitud de madurez) y la mortalidad natural. Posteriormente Charnov (1993) realizó una extensiva revisión de estas invariantes de la historia de vida, entendiéndose por invariante alguna característica demográfica que no cambia a través de las especies y poblaciones, las cuales carecen de unidades de medida, denominádolas como las invariantes de Beverton y Holt, que han servido como herramientas prácticas para caracterizar la dinámica y la historia de vida de una población poco estudiada e incluso para estimar máximo rendimiento sostenible y la tasa de mortalidad que produce el máximo rendimiento (Beddington \& Kirkwood 2005).

Las tres invariantes de la historia de vida tratadas por Charnov (1993) son: (i) el producto entre la mortalidad natural $(M)$ y la edad de primera madurez $\left(t_{m}\right)$; (ii) la razón entre $M$ y el coeficiente de crecimiento del modelo de von Bertalanffy (K); (iii) la razón entre la longitud de madurez $\left(L_{m}\right)$ y la longitud asintótica $\left(L_{\infty}\right)$. Jensen (1996) obtiene los valores de las invariantes maximizando la función de fecundidad y asumiendo que los organismos crecen de acuerdo al modelo de von Bertalanffy, siendo estos:

$$
\begin{aligned}
& M t_{m}=1,65 \\
& \frac{M}{K}=1,5 \\
& \frac{L_{m}}{L_{\infty}}=0,66
\end{aligned}
$$

La existencia de estas tres invarianzas ha sido investigada principalmente en peces (Beverton \& Holt 1959), para la última invariante, estos autores entregan valores entre 0,4 y 0,8 e indican que ciertos valores se mantienen a nivel de taxón. En general cuando se estima la invariante $L_{m} / L_{\infty}$, algunos obtienen un promedio, e.g., Blanck et al. (2007) promedió para peces de agua dulce y obtiene un valor de 0,49, Vollestad \& L’Abée-Lund (1994) estima un valor de 0,728 para 30 poblaciones de Salvelinus alpinus. Cuando se calcula el promedio no se consideran las características de cada estudio en el sentido de la exactitud de las estimaciones de los parámetros, en el caso de la estimación de la longitud de madurez, ésta se puede realizar por medio de métodos macro o microscópico lo que obviamente influye en los resultados. Por otra parte, se promedia considerando estudios realizados en distintos años, no evaluando el efecto que podría tener la pesca en los parámetros de la historia de vida de recursos explotados.

Resultados publicados sobre $L_{m}$ y $L_{\infty}$ en peces de la costa de Chile, son adecuados para la realización de un meta-análisis. Según Glass (1976 fide Rosemberg et al. 2000), que incorpora el concepto de meta-análisis, indica que es el análisis estadístico de una colección de estudios individuales con el propósito de integrar cuantitativamente los resultados y se calcula un valor medio global de la cantidad medida, además permite analizar la heterogeneidad de ésta entre los diferentes estudios. Para ello se requiere definir una medida de los resultados que sea homogénea en todos los estudios, en este caso corresponde al tamaño del efecto, que representa la magnitud en que se manifiesta el fenómeno en cuestión en cada estudio empírico, siendo para este estudio, la misma invariante.

Por lo anterior, se evaluó el valor de la invariante $L_{m}$ / $L_{\infty}$ para peces de la costa de Chile y se comparó con lo propuesto por Jensen (1996), planteándose los siguientes objetivos, estimar el tamaño del efecto medio, determinar la existencia de heterogeneidad en torno al tamaño del efecto entre los estudios y determinar las variables que pueden estar moderando el tamaño del efecto.

\section{Material y MÉtodos}

Se revisó la información disponible y los resultados de diversos proyectos de investigación en recursos pesqueros a través del Fondo de Investigación Pesquera (FIP) en Chile junto con los resultados de búsqueda en Internet de información disponible en trabajos científicos (e.g., longitud madurez, longitud asintótica, von Bertalanffy peces Chile). Los estudios que estiman ambos parámetros $\left(L_{m} \mathrm{y} L_{\infty}\right)$ son escasos por lo que se tuvo que complementar considerando estimaciones de $L_{m}$ o $L_{\infty}$ por separado de otros estudios, teniendo en cuenta la zona geográfica y el año en que se tomó la muestra. De los trabajos recopilados se obtuvo la siguiente información ${ }^{1}$ : fuente, familia, especie, año muestreo, zona geográfica, sexo, $L_{\infty}$, error estándar de $L_{\infty}, L_{m}$ e intervalos de confianza (IC) de $L_{m}$.

La metodología que se utilizó para realizar el metaanálisis se basa en Rosenberg et al. (2000, 2004) y Borenstein et al. (2009), la cual se encuentra implementada en el software MetaWin (Rosenberg et al. 2000). Como tamaño del efecto se consideró el valor de la invariante estimada para cada especie:

${ }^{1}$ La base de datos empleada se encuentra disponible vía correo electrónico con el autor, maraya@uantof.cl 


$$
I_{i k}=\left(\frac{L_{m}}{L_{\infty}}\right)_{i k}
$$

donde $\left(L_{m} / L_{\infty}\right)_{i k}$ es la invariante correspondiente a la especie $i$ del estudio $k$; la varianza de la invariante para la especie $i$ del estudio $k$ se obtuvo mediante remuestreo en el cual para $L_{m}$ se consideró una distribución triangular debido a la incerteza de la información y para $L_{\infty}$ una distribución normal. Los límites de la distribución triangular y desviación estándar para la distribución normal se obtuvieron de los mismos trabajos.

Una vez que el tamaño del efecto y la varianza son estimados para cada estudio, el siguiente paso es resumir los resultados individuales para entender el efecto total, donde los estudios son ponderados dependiendo de su exactitud, i.e., estudios con baja varianza presentan un mayor peso que con una varianza mayor; el peso para cada estudio corresponde a la inversa de su varianza:

$$
w_{i k}=\frac{1}{s_{i k}^{2}}
$$

En meta-análisis un modelo de efectos fijos asume que hay un solo valor del tamaño del efecto, el cual corresponde a un promedio ponderado de los tamaños de los efectos individuales y se estima por:

$$
\bar{I}=\frac{\sum w_{i k} I_{i k}}{\sum w_{i k}}
$$

con varianza:

$$
s_{\bar{I}}^{2}=\frac{1}{\sum w_{i k}}
$$

$\mp$ corresponde al efecto promedio para todos los estudios y el IC se estimó mediante bootstrap.

Para probar si el tamaño del efecto es homogéneo, es decir, provienen de una sola población, se usó el estadístico $Q$ (una forma de suma de cuadrado ponderado) (Huedo-Medina et al. 2006). La heterogeneidad total se define por:

$$
Q_{T}=\sum w_{i k}\left(I_{i k}-\bar{I}\right)^{2}
$$

$Q_{T}$ se prueba contra la distribución $\chi^{2}$ con n-1 grados libertad. Esta prueba evalúa si el tamaño de los efectos para todos los estudios es igual; un resultado significativo indica que la varianza es más grande que el esperado debido a un error de muestreo. El cálculo asume que todos los estudios provienen de una sola muestra homogénea. A menudo la variación en el $I$ puede ser explicada por variables adicionales (e.g., taxón, año de muestreo).

Si el resultado de la prueba indica que no es significativa, se puede obtener un sólo valor de $I$. Cuando no se cumple el supuesto del tamaño del efecto homogéneo, se debe incorporar la heterogeneidad entre el tamaño de los efectos en un modelo de efectos aleatorios.

En el modelo de efectos aleatorios, se asumió que $I_{i k}$ no es fijo entre familias (Longhurst \& Pauly 1987), por lo que, la variabilidad total observada en el tamaño del efecto $I_{i k}$ se divide en el error de muestreo y una componente aleatoria de variación en el tamaño del efecto entre estudios $\left(\sigma_{\text {entre }}^{2}\right)$ (Borenstein et al. 2009). Esta varianza se utilizó para calcular el peso del modelo de efectos aleatorios.

$$
w_{i k(\text { aleat })}=\frac{1}{s_{i k}^{2}+\sigma_{\text {entre }}^{2}}
$$

Este ponderador se utilizó en el modelo de efectos aleatorios de las ecuaciones (6) y (8) y la estimación de se realizó mediante:

$$
\sigma_{\text {entre }}^{2}=\frac{Q_{E}-(n-m)}{\sum_{j=1}^{m}\left[\sum_{i=1}^{k_{j}} w_{i j} \frac{\sum_{i=1}^{k_{j}} w_{i j}^{2}}{\sum_{i=1}^{k_{j}} w_{i j}}\right]}
$$

donde $Q_{E}$ es la heterogeneidad del error residual del modelo de efectos fijos; $n$ es el número total de casos, $m$ es el número de familias consideradas, $k_{j}$ es el número de casos en el $j^{h}$ grupo; $w_{i j}$ es el peso estimado en el modelo efectos fijos del $i^{h}$ caso en la $j^{h}$ familia.

\section{Resultados}

Se recopilaron 287 casos, entendiendo como caso, especie y uno o dos valores de $L_{m}$ y $L_{\infty}$. De estos, sólo se 
obtuvieron 148 valores de la invariante $L_{m} / L_{\infty}$ para 14 especies pertenecientes a diez familias de peces y cuyos años de muestreo se encuentran entre 1964 y 2006, oscilando los valores entre 0,26 y 0,8. En la distribución de frecuencia de los valores de la invariante (Fig. 1), se encontraron dos modas, la primera entre 0,56 y 0,62 y la segunda entre 0,74 y 0,76 . En esta misma figura se aprecia que individuos de una misma familia abarcan un amplio rango del valor de la invariante, e.g., Merlucciidae entre 0,46 y 0,80; Clupeidae entre 0,50-0,62.

La media sin ponderar de los 148 datos de la invariante $L_{m} / L_{\infty}$ fue de 0,597 con un IC de 0,58-0,61, cuyo intervalo no abarca el valor de 0,66. Al estimar la invariante por familia (Tabla 1, Fig. 2) sólo tres, Nototheniidae, Scombridae y Trachichthyidae presentan el valor 0,66 en el IC.

El resultado de la aplicación del modelo de efectos fijos entregó un valor de la media ponderada de 0,71 y en la prueba de heterogeneidad se rechaza la hipótesis nula de homogeneidad entre los tamaño de los efectos $\left(Q_{T}=\right.$ $313,03, P<0,05)$ (Tabla 2), es decir existen diferencias por una variable que estaría influyendo en los resultados. La aplicación del modelo de efectos aleatorios se realizó a nivel de familia entregando un valor de la media ponderada de 0,61 (Tabla 2).

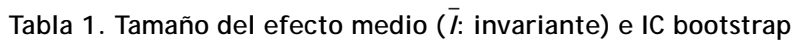
para 9 familias de peces. En negrita las IC que abarcan el valor de $\mathbf{0 , 6 6}$. No se incluye Sciaenidae por tener sólo un valor / Mean effect size ( $\overline{\mathrm{I}}$ : invariant) and confidence intervals bootstrap for 9 fish families. In bold IC covering 0.66 value. Sciaenidae not incluyed because has one value

\begin{tabular}{lrrr}
\hline Familia & Número de casos & $\bar{I}$ & IC bootstrap \\
\hline Bramidae & 3 & 0,69 & $0,68-0,70$ \\
Carangidae & 16 & 0,48 & $0,43-0,53$ \\
Clupeidae & 13 & 0,56 & $0,54-0,58$ \\
Engraulidae & 15 & 0,52 & $0,47-0,56$ \\
Merlucciidae & 60 & 0,63 & $0,61-0,65$ \\
Nototheniidae & 9 & 0,65 & $\mathbf{0 , 6 0}-\mathbf{0 , 7 0}$ \\
Ophidiidae & 23 & 0,61 & $0,58-0,63$ \\
Scombridae & 4 & 0,68 & $\mathbf{0 , 6 4}-\mathbf{0 , 7 2}$ \\
Trachichthyidae & 4 & 0,69 & $\mathbf{0 , 6 5}-\mathbf{0 , 7 3}$ \\
\hline
\end{tabular}

Tabla 2. Resultados de la aplicación de los modelos de efectos fijos y aleatorios / Application results of fixed and random effects models

\begin{tabular}{lcc}
\hline Estadísticos & $\begin{array}{c}\text { Modelo } \\
\text { Efectos Fijos }\end{array}$ & $\begin{array}{c}\text { Modelo } \\
\text { Efectos Aleatorios }\end{array}$ \\
\hline Número de casos & 148 & 148 \\
Tamaño del efecto medio ponderado & 0,71 & 0,61 \\
IC bootstrap & $0,68-0,73$ & $0,59-0,63$ \\
$\sigma_{\text {entre }}^{2}$ & & 0,0071 \\
Qtotal & 5448,83 & 313,03 \\
& $(P<0,05)$ & $(P<0,05)$ \\
\hline
\end{tabular}

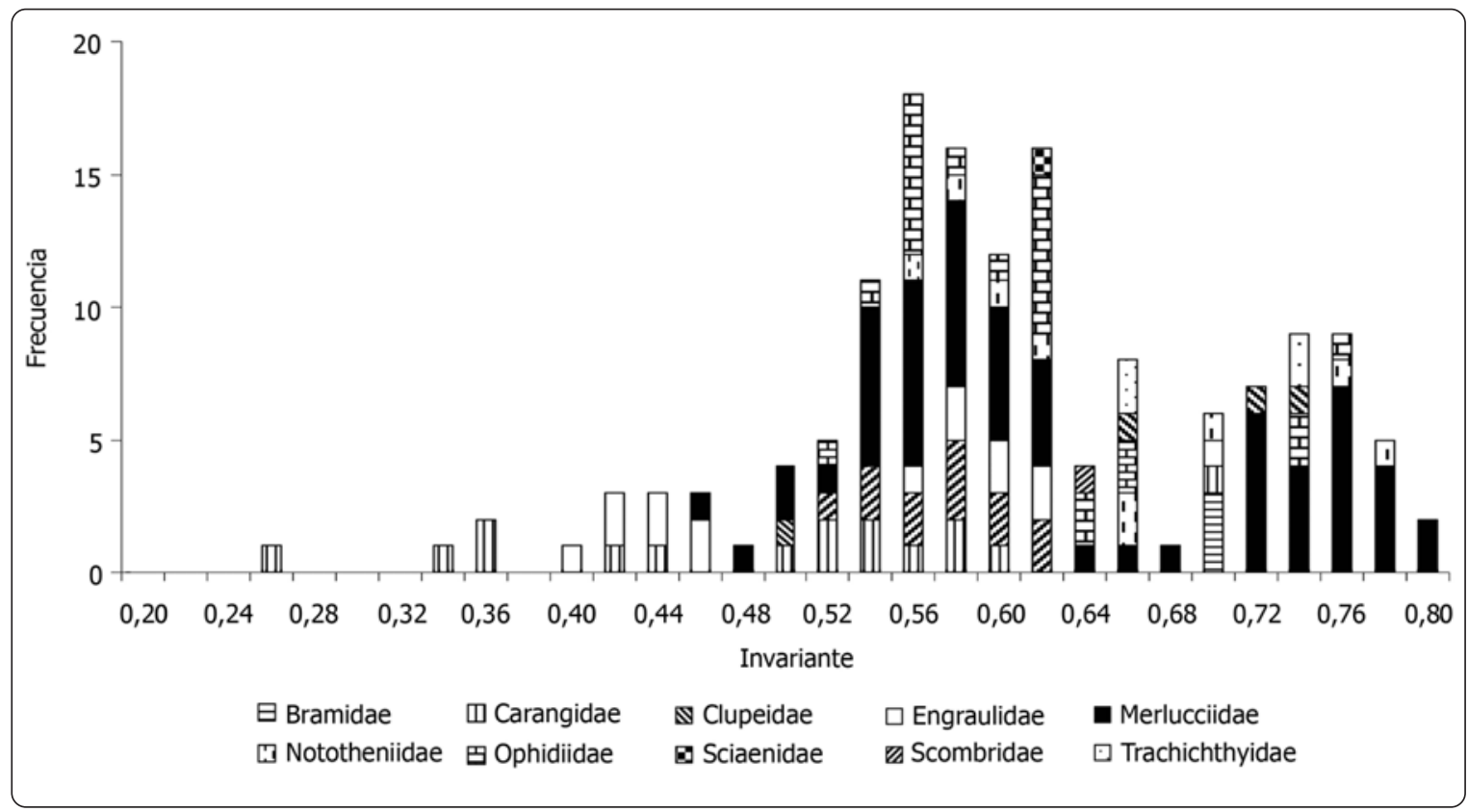

Figura 1. Distribución de frecuencia de la invariante $\mathrm{L}_{\mathrm{m}} / \mathrm{L}_{\infty}$ para diez familias de peces / Frequency distribution of the invariant $L_{m} / L_{\infty}$ for ten fish families 


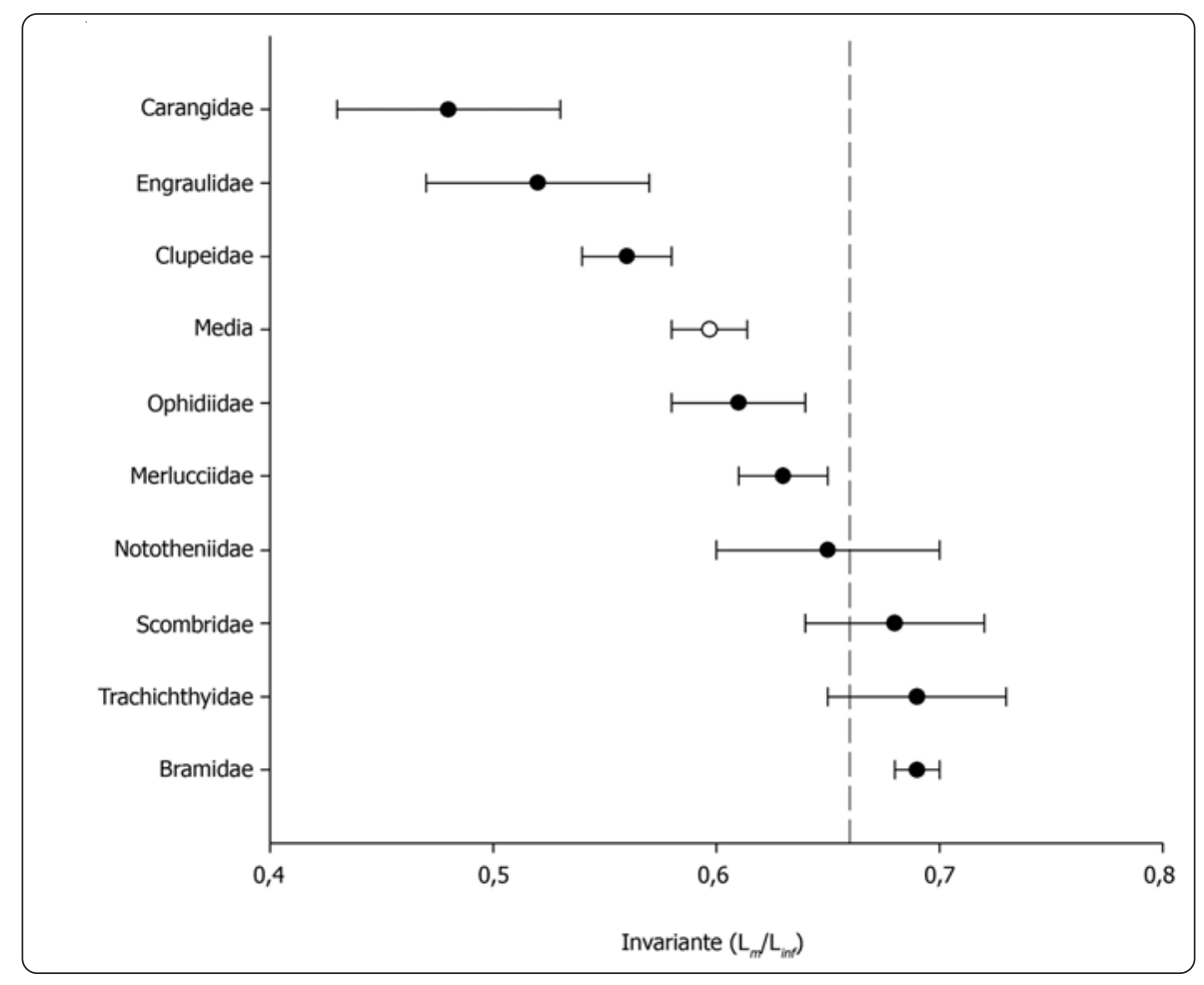

Figura 2. Media sin ponderar e IC de la invariante por familia. Cada punto representa el tamaño del efecto medio para peces de una familia determinada sobre la que se recopiló información. Línea discontinua corresponde al valor 0,66 / Average and confidence interval of the invariant by family. Each point represents the mean effect size for fish of a given family on which information was collected. Dashed line corresponds to the value of 0.66

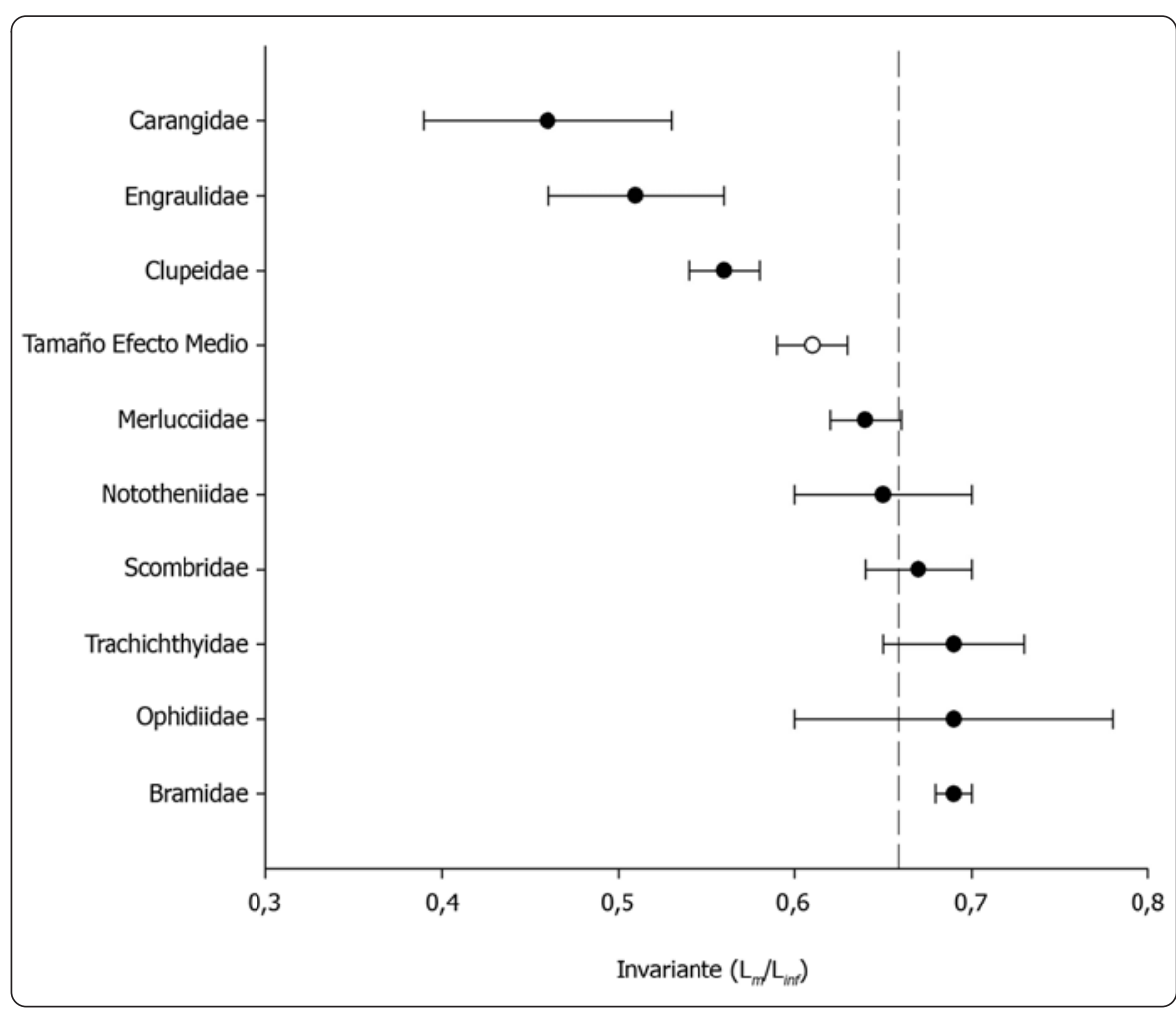

Figura 3. Invariante $L_{m} / L_{\infty}$ (tamaño del efecto) e IC por familia, estimado en base al modelo de efectos aleatorios. Cada punto representa el tamaño del efecto medio para peces de una familia determinada sobre la que se recopiló información. El tamaño del efecto medio representa el valor medio ponderado. Línea discontinua corresponde al valor $\mathbf{0 , 6 6} /$ Invariant $\mathrm{L}_{m} / \mathrm{L}_{\infty}$ (effect size) and confidence intervals by family, based on the estimated random effects model. Each point represents the mean effect size for fish of a given family on which information was collected. The average effect size represents the weighted average overall. Dashed line corresponds to the value 0.66 
Según los IC estimados con el modelo de efectos aleatorios, cinco familias contienen el valor 0,66 , Merlucciidae $(0,64)$, Nototheniidae $(0,65)$, Ophidiidae $(0,69)$, Scombridae $(0,67)$ y Trachichthyidae $(0,69)$. La media para las familias Carangidae, Clupeidae y Engraulidae fueron 0,46, 0,56 y 0,51, respectivamente, por debajo del valor de la invariante 0,66 . La media de la familia Bramidae fue de 0,69 (Fig. 3)

\section{Discusión}

En el ámbito pesquero se ha reconocido la necesidad de realizar análisis comparativos de estudios biológicos (Pauly 1980, Myers et al. 2001, Reynolds et al. 2005), esto debido a la escasez de series de tiempo para evaluar el efecto de los cambios ambientales y de explotación en las poblaciones de peces (Myers \& Mertz 1998). En tal sentido los resultados del presente estudio confirman el cuidado que hay que tener en el uso de invariantes cuando se realizan estimaciones rápidas de otros parámetros.

Como todo método de inferencia el meta-análisis tiene sus problemas y limitaciones. Uno de los problemas sería el que se deriva del procedimiento al combinar datos de múltiples estudios que pudieran tener diferencias en la metodología utilizada para la estimación de los parámetros, e.g., estimación de $L_{m}$. Algunos estudios utilizan criterios macroscópicos y otros microscópicos que influyen en la precisión del parámetro, se suma a ello que en la mayoría de los estudios revisados no hay resultados de algún estadístico de variabilidad o de error. Por otro lado, para determinar el parámetro $L_{\infty}$, en una misma especie, se pueden utilizar escamas u otolitos para estimar la edad, en el ajuste del modelo de von Bertalanffy se encuentran diferencias si se utiliza información retrocalculada o de la edad actual y la mayoría de los estudios carecen de la validación del método para estimar edad y no se entrega una valor de variabilidad o error de los parámetros. Otra situación que influye en el valor de los parámetros corresponde al tamaño de la muestra y al rango de longitud utilizado, faltando los individuos jóvenes o bien los más viejos.

Estudios han demostrado que los individuos de poblaciones explotadas incrementan su eficacia biológica mediante el aumento de la tasa de crecimiento y disminuyen la edad y tamaño a la primera madurez, por lo que los parámetros de la historia de vida cambian en respuesta a la pesca, estos cambios son el resultado de la plasticidad fenotípica, pero en el largo plazo la presión selectiva de la pesca puede llevar a cambios genéticos (Rochet 1998, Jennings et al. 1999, Shin et al. 2005, Sharpe \& Hendry 2009). La información del efecto que puede estar ocasionando la pesca, en los parámetros de la historia de vida de las poblaciones de nuestras costas, es escasa y fragmentada, aunque se debiera esperar que la invariante por familia no cambie debido a la disminución de ambas longitudes ante el efecto de la pesca, siempre y cuando los cambios se produzcan a la misma escala temporal.

Pauly (1994) plantea que una explicación para la invariante $L_{m} / L_{\infty}$ es el concepto de "drenaje reproductivo", es decir, que los peces una vez que han alcanzado la madurez sexual, presentan una disminución en su tasa de crecimiento para destinar energía a la producción de material gonadal. Otra hipótesis que explica el desove de los peces a una fracción predecible de su tamaño asintótico, sería por el modelo de crecimiento metabólico (Pauly 1984, Longhurst \& Pauly 1987), ya que los peces cuando alcanzan el peso asintótico carecen de energía disponible que podría ser destinada al fortalecimiento de las gónadas, por lo que debe reproducirse a un tamaño menor. Es así que el valor de $L_{m} / L_{\infty}$ suele ser menor en peces grandes y superior en peces pequeños y aproximadamente constante a nivel de familias en peces de longitudes similares. Jensen (1996) obtiene las invariantes al maximizar la función de fecundidad, la cual optimiza el balance entre sobrevivencia y fecundidad, sin embargo Charnov (1993) propone una explicación para las invariantes de Beverton \& Holt (1959) desde un punto de vista evolutivo al maximizar la eficacia biológica de la población. Charnov (1993) propone que la razón entre $L_{m} / L_{\infty}$ es totalmente dependiente del parámetro que compensa los parámetros $L_{\infty}$ y $\mathrm{K}$ (parámetro denominado 'h' en Charnov 1993). Por otra parte, el parámetro h parece ser constante solo dentro de grupos taxonómicos (e.g., Clupleiformes) y no a través de todas las especies de peces, como se podría derivar desde la teoría de Jensen (1996).

Las estimaciones de las invariantes por familia obtenidas en el presente estudio, pueden servir para estimar una variable a partir de la otra cuando la información es escasa sobre todo en poblaciones explotadas. Se desprende que el valor teórico de la invariante $L_{m} / L_{\infty}=0,66$ propuesto por Jensen (1996) no es generalizable para todas las especies de peces, considerando la variabilidad e incerteza que presenta la información. El tamaño del efecto medio ponderado se estimó en 0,61 cuyo IC no incluye en su recorrido el valor de la invariante 0,66 , esto por la heterogeneidad de la información que resultó significativa. Dado el resultado de heterogeneidad del tamaño del efecto se puede indicar que la variable que modera los resultados corresponde al taxón familia (Tabla 3). Esto concuerda con lo indicado por Beverton \& Holt (1959) y Longhurst \& Pauly (1987) en que la invariante $L_{m} / L_{\infty}$ presentaría valores similares a 
Tabla 3. Tamaño del efecto medio (Ī) e IC bootstrap por familia estimado mediante el modelo de efectos aleatorios. En negrita IC de valor $\mathbf{0 , 6 6}$. No se incluye Sciaenidae por tener sólo un valor de la invariante / Mean effect size (I) and confidence intervals bootstrap by family estimated by random effects model. In bold IC containing 0.66 values. Sciaenidae not incluyed because has one value for the invariant

\begin{tabular}{lrrr}
\hline Familia & Número de casos & $\bar{I}$ & IC bootstrap \\
\hline Bramidae & 3 & 0,69 & $0,68-0,70$ \\
Carangidae & 16 & 0,46 & $0,39-0,52$ \\
Clupeidae & 13 & 0,56 & $0,54-0,57$ \\
Engraulidae & 15 & 0,51 & $0,46-0,57$ \\
Merlucciidae & 60 & 0,64 & $\mathbf{0 , 6 2}-\mathbf{0 , 6 7}$ \\
Nototheniidae & 9 & 0,65 & $\mathbf{0 , 6 0}-\mathbf{0 , 7 0}$ \\
Ophidiidae & 23 & 0,69 & $\mathbf{0 , 6 0}-\mathbf{0 , 7 8}$ \\
Scombridae & 4 & 0,67 & $\mathbf{0 , 6 4}-\mathbf{0 , 7 1}$ \\
Trachichthyidae & 4 & 0,69 & $\mathbf{0 , 6 5}-\mathbf{0 , 7 3}$ \\
\hline
\end{tabular}

nivel de familia e incluso de orden, destacando 0,75 para clupeidos, que no concuerda con el valor obtenido en el presente estudio de 0,56 .

\section{Agradecimientos}

Los autores agradecen los comentarios realizados por dos revisores anónimos y uno de los editores de la revista.

\section{LITERATURA CITADA}

Beddington JR \& GP Kirkwood. 2005. The estimation of potential yield and stock status using life-history parameters. Philosophical Transactions of the Royal Society B 360: 163-170.

Beverton RJH \& SJ Holt. 1959. A review of the lifespans and mortality rates of fish in nature, and their relation to growth and other physiological characteristics. En: Wolstenholme GEW \& M O'Connor (eds). CIBA Foundation Symposium (Colloquia on Ageing). 5. The lifespan of animals, pp. 142180. J \& A Churchill, London.

Blanck A, PA Tedesco \& N Lamouroux. 2007. Relationships between life-history strategies of European freshwater fish species and their habitat preferences. Freshwater Biology 52: 843-859.

Borenstein M, LV Hedges, JPT Higgins \& HR Rothstein. 2009. Introduction to meta-analysis, 450 pp. John Wiley \& Sons, Chichester.

Charnov EL. 1993. Life history invariants. Some explorations of symmetry in evolutionary ecology, $184 \mathrm{pp}$. Oxford University Press, Oxford.

Huedo-Medina TB, J Sánchez-Meca, J Botella \& F MarínMartínez. 2006. Assessing heterogeneity in meta-analysis: $Q$ Statistic or $l^{2}$ Index?. Psychological Methods 11: 193-206.
Jennings S, SPR Greenstreet \& JD Reynolds. 1999. Structural change in an exploited fish community: A consequence of differential fishing effects on species with contrasting life histories. Journal of Animal Ecology 68: 617-627.

Jensen AL. 1996. Beverton and Holt life history invariants result from optimal trade-off of reproduction and survival. Canadian Journal of Fisheries and Aquatic Sciences 53: 820-822.

Longhurst AR \& D Pauly. 1987. Ecology of tropical oceans, 407 pp. Academic Press, San Diego.

Myers RA \& G Mertz. 1998. Reducing uncertainty in the biological basis of fisheries management by meta-analysis of data from many populations: a synthesis. Fisheries Research 37: 51-60.

Myers RA, BR MacKenzie, KG Bowen \& NJ Barrowman. 2001. What is the carrying capacity for fish in the ocean? A meta-analysis of population dynamics of North Atlantic cod. Canadian Journal of Fisheries and Aquatic Sciences 58: 1464-1476.

Pauly D. 1980. On the interrelationships between natural mortality, growth parameters, and mean environmental temperature in 175 fish stocks. ICES Journal of Marine Science 39: 175-192.

Pauly D. 1984. A mechanism for the juvenile-to-adult transition in fishes. Journal du Conseil International pour l'Exploration de la Mer 41: 280-284.

Pauly D. 1994. On the sex of fish and the gender of scientist: essays in fisheries science. Chapman \& Hall, London, 250 p.

Reynolds JD, NK Dulvy, NB Goodwin \& JA Hutchings. 2005. Biology of extinction risk in marine fishes. Proceedings of the Royal Society B 272: 2337-2344.

Rochet MJ. 1998. Short-term effects of fishing on life history traits of fishes. ICES Journal of Marine Science 55: 371391.

Rosenberg MS, DC Adams \& J Gurevitch. 2000. MetaWin: Statistical software for meta-analysis, version 2.0. Sinauer Associates, Sunderland. [CD-ROM]

Rosenberg MS, KA Garret, Z Su \& RL Bowden. 2004. Metaanalysis in plant pathology: Synthesizing research results. Phytopathology 94: 1013-1017.

Sharpe DMT \& AP Hendry. 2009. Life history change in commercially exploited fish stocks: an analysis of trends across studies. Evolutionary Applications 2: 260-275.

Shin Y-J, M-J Rochet, S Jennings, JG Field \& H Gislason. 2005. Using size-based indicators to evaluate the ecosystem effects of fishing. ICES Journal of Marine Science 62: 384-396.

Vollestad LA \& JH L'Abée-Lund. 1994. Evolution of the life history of Arctic charr Salvelinus alpinus. Evolutionary Ecology 8: 315-327. 